\title{
POTENTIALS EVALUATION FOR INSTANT USABILITY OF SMARTPHONE APPS IN HEALTH AND SPORTS ENDURANCE ACTIVITY TRACKING
}

\author{
Hans Weghorn \\ Baden-Wuerttemberg Cooperative State University Stuttgart \\ Kronenstrasse 53A, 70174 Stuttgart, Germany
}

\begin{abstract}
Systematic control of physical workouts is required for achieving good curing or caring effects in health activities as well as for sustaining or even improving fitness level in non-elite and professional sports. For this purpose, respectively wearable medical monitors as also a wide range of personal sports computers were commonly in use since longer time. With the overwhelming evolvement of the smartphone market, health and sports monitoring was identified also as prospective working field for app developers. Accordingly, numerous smartphone apps are offered meanwhile for endurance training, many of them even as so-called freeware. An investigation is presented here about the required efforts in setup and in daily use as also about the reliability of such smartphone monitoring, taking especially into account the needs of possible elderly users. Furthermore, it is critically discussed in this work, whether smartphone apps really can replace the instant and seamless use mode of traditional sports computers.
\end{abstract}

\section{KEYWORDS}

Ubiquitous Health Monitoring, Sports Tracking Tools, Personalized Mobility, Elderly Care, Smartphone Training Apps

\section{INTRODUCTION}

Regular, weekly physical training preserves good health up to high living ages. This was repeatedly shown in medical studies. For instance, it has been particularly validated, that weekly brisk walking can reduce stroke risk for people at ages even above 70 (Jefferis et al., 2014). Numerous investigations report also positive effects on various other pathological conditions (Law et al., 1991), but especially diminishing stroke risks was repeatedly under research for men (Abbott et al., 1994) and women (Oguma, Shinoda-Tagawa, 2004).

Endurance recreation sports like nordic walking or jogging can be performed easily without special facilities and without registering with any club or community. The particular benefit of both activities was investigated also early (Suter et al., 1994) (Church, 2010), but it was found later that too high training loads can be worse than avoiding physical exercises at all (Schnohr et al., 2015).

Accordingly, workout control appears mandatory, because the demand level has to be kept within certain limits for gaining positive effects, but also for preventing problems caused by too intense load (Suter et al., 1994) (Schnohr et al., 2015). Also for this reason, tiny sports computers were broadly commercialized since fairly long time. The modern version are powerful watch-like units with built-in GPS sensing and expandability by RF-linked body sensors, e.g. for registering also heart rate or food stride rate.

A converging commercial technology came up during the recent decades in the form of smartphones, which are meanwhile even equipped with various sensors, e.g., for detecting humidity and temperature of their environment, lighting conditions and others. Smartphones can trace their own movement by built-in acceleration sensors (Valentin, Howard, 2013), modern smartphones are typically equipped also with GPS positioning and network localization modes for improving the detected location. Furthermore, Bluetooth communication allows them to interlink with body sensors like chest straps for heart rate monitoring. 
This set of technical features manifests a perfect base for a use of smartphones in fitness and sports tracking, which would help to overcome the need for other specialized devices like tiny sports computers. For the matter of completeness, it should be mentioned here, that other upcoming product segments like smart watches and fitness bracelets also aim to serve this application field (Alsulami, Atkins, 2016), but the severe limitations of such devices have been investigated and published recently before (Weghorn, 2019). The investigation reported here addresses the question, whether smartphones with appropriate application software tools are available yet, which can replace the established sports computers with the same degree of seamless and instant handling in daily endurance workouts.

\section{EXPERIMENTAL EVALUATION OF RELIABILITY AND USABILITY}

\subsection{Field Testing Environment and Selected Devices}

As experimental tracking devices a set of Android smartphones was selected from the middle price segment. Android phones were chosen, because this operating system has got the highest market share (statcounter, 2019) and stands therefore for the most typical device and handling surface, but the phones came from different vendors and had different major versions of Android for exploiting also the impact of the evolvement of the operating system. Table 1 lists these units, but for legal reasons neither brands nor particular device models are identified here.

Table 1. Devices used for tracking in experiments

\begin{tabular}{llll}
\hline Device & Vendor & OS level & Device description \\
\hline $\mathrm{A}$ & $\mathrm{X}$ & Android 5 & Multimedia consumer device \\
$\mathrm{B}$ & $\mathrm{Y}$ & Android 6 & Robust outdoor smartphone \\
$\mathrm{C}$ & $\mathrm{Z}$ & Android 7 & Universal standard device \\
$\mathrm{T}$ & $\mathrm{G}$ & proprietary & Triathlon sports watch computer \\
\hline
\end{tabular}

As established reference for instant use in tracking, the triathlon sports watch $\mathrm{T}$ (Figure 1b) manufactured by a reputed vendor, who has got long-term experience in this device market, has been used throughout the experimental series in parallel to A, B and C. This sports computer provides motion tracking through GPS and built-in acceleration sensors, as also additional optional monitoring through RF-coupled sensors for heart rate, pedal turn, foot strides and further more. After switching on, it can be used instantly by a single hard button press for initiating a workout tracking. If precise GPS trace is desired, start of workout better is delayed until sufficient signal receipt quality is reached and indicated.

All measurements were performed on a public course of $5 \mathrm{kms}$ (Figure 1d), which was installed in 2018 as part of a health and sports supporting project in a joint venture between two neighbor cities and a sports club with long tradition, all located next to the Danube river in central Europe. This project is described on the web site www.donaurunning.de, where also recent results and long term of use statistics can be displayed.

Users of this parkour are automatically monitored during their running and walking activities through the four RF gates (Figure 1a), when they are wearing their particular ID chip (Figure 1c). All conducted laps are counted and can be inspected on the project web site. The official length of the track is 4.99 kilometers, but the project leader reminded in a private communication in December 2018, that the precise measure is more likely 4.98 kilometers. This is a quite typical distance for any walking workout, and a running round of roughly 30 minutes duration stands also for a basic training effort (Suter et al., 1994). The permanently available course represents a reproducible experimental field route for the investigation here, and it maps well the spirit of endurance work-outs in healthy living style and personal sports. 

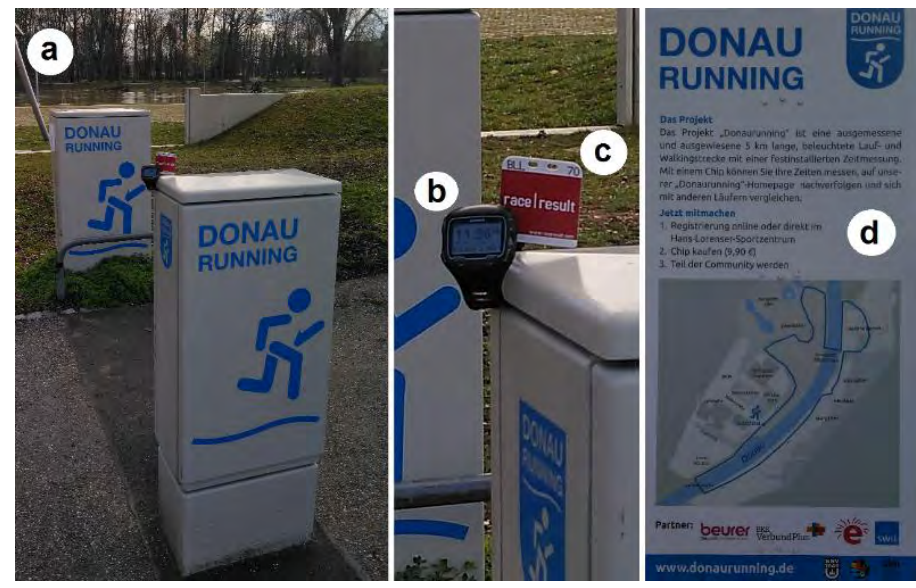

Figure 1. The running and walking parkour round, which was used for the tests, has got a length of 5 kilometers (d) and is equipped with four gates (a), through which sports activity is monitored automatically for people owning and wearing the corresponding RF ID chip (c)

\subsection{Measurement Series on Precision of Movement Tracking}

The original plan for the experiments was to first select a feasible smartphone fitness app and perform the following precision tests on base of this. It quickly turned out, that none of the available fitness apps provided such a seamless handling opportunity, so that this part of the investigation could be focused solely on the question of tracking precision. In the end, an app was found, which was intended more as general tracking tool, e.g. in car driving, for determining current velocity, elapsed time and distances and other values. For this, it uses a rather simple-styled display arrangement with big letters and minimal input buttons (Figure 2).

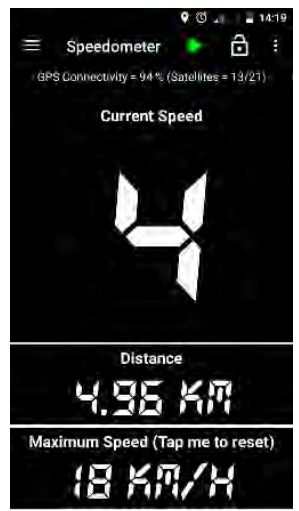

Figure 2. Screenshot of the simple app, which was used for validating the position tracking capabilities of the smartphones. The capture was taken after completing the Danube running round, it is clearly readable, the tracking screen consists of few UI elements only, and is primarily designed as assistive speed control during car driving

Furthermore, few GPS signal analysis apps were found for controlling the feasibility of the system platforms for detecting and tracing geographic location (GPS satellite positioning testing app is shown in Figure 4). In this configuration, the route length measurements in Tab. 2 have been recorded at varying jogging speeds.

Table 2. Measured distances in jogging around the Danube running loop

\begin{tabular}{cccc}
\hline Device & \# of laps & Moving speed & Average distance and statistical variation \\
\hline A & 5 & $12.6 \mathrm{~km} / \mathrm{h}+/-0.68 \mathrm{~km} / \mathrm{h}$ & $4,850 \mathrm{kms}+/-0,0363 \mathrm{kms}$ \\
$\mathrm{B}$ & 5 & $12.2 \mathrm{~km} / \mathrm{h}+/-0.42 \mathrm{~km} / \mathrm{h}$ & $4,883 \mathrm{kms}+/-0,0221 \mathrm{kms}$ \\
$\mathrm{T}$ & 20 & $12.4 \mathrm{~km} / \mathrm{h}+/-0.69 \mathrm{~km} / \mathrm{h}$ & $4,883 \mathrm{kms}+/-0,0206 \mathrm{kms}$ \\
\hline
\end{tabular}


As seen here, the average errors in distance tracking of the $4.99 \mathrm{kms}$ path range in the order of 100 meters absolutely or $2 \%$ relatively. Though the measures arise strictly too low, this all appears acceptable for tracing workouts in health or non-elite sports. There is almost no difference between the tiny sports watch and the bigger smart phone devices. All devices use GPS, which is assumed due to its low sampling speed to have intrinsic problems of reduced distance measures in, e.g., tight curves, which are present in the workout parkour also at several turning points.

Furthermore, the route path on the Danube running loop is not exactly or strictly defined, the walking paths have varying widths up to several meters. In the experiments above, the shortest connections between turning points were followed strictly, inner curve radiuses were used whenever possible, but the experimenters stayed always on the lanes.

For validating GPS errors, which are presumably induced by a low sampling rate especially in turns, the parkour was completed in the next tests at slower speed with even slower motion through all the tight curves. First, the shortest walking path was traced in a single shot experiment, and next, a path through the walking round was chosen with extended swings, but still remaining on the walking lanes. Table 3 list comparatively the corresponding results.

Table 3. Test rounds with slow motion especially in tight curves

\begin{tabular}{llll}
\hline Device & Course & Curve routing & Measured distance \\
\hline $\mathrm{B}$ & Shortest & Innermost & $4,924 \mathrm{kms}$ \\
$\mathrm{T}$ & Shortest & Innermost & $4,879 \mathrm{kms}$ \\
$\mathrm{B}$ & Wide swing & Outer & $4,948 \mathrm{kms}$ \\
$\mathrm{T}$ & Wide swing & Outer & $4,952 \mathrm{kms}$ \\
\hline
\end{tabular}

These measurements do support the theory about the GPS sampling error problem, but show also the imprecision of the route itself. These distance values are closer to the official measure of the parkour, though Table 2 has shown already the capability of the smartphone devices for sufficient precision in route tracking.

Device A was working during the first four experiments well, but then suddenly it lacked completely of any localization capabilities. A lot of reboots and configuration checks couldn't fix the problem for many days, several experiments on workout tracking failed for this reason. In the end, the device started suddenly working again, when it was linked permanently to an Internet connection and was not moved physically until the GPS test tools aligned to the satellite signals sufficiently. This observation is interpreted as showing a fundamental problem with the operating system version and its localization method, especially with the internal improvement functionality of localization within the operating system that bases on additional information aside GPS, like e.g. surveillance of close-by WLAN networks. With device B and its newer OS version, such issues haven't been observed. Up to the project state now, experiments with device $\mathrm{C}$ couldn't be performed in a systematic way yet.

Another side observation concerns the handling of GPS localization in the devices in general. T activates this method immediately after being switched on. Localization reaches sufficient precision in the order of a minute, while with the smartpones it may take considerably longer. This is invoked by the powersaving approach of activating and de-activating the GPS module in smartphones on demand, which incurs a longer waiting period for GPS after a re-launch of the tracking app, despite the fact that it had been used just shortly before. Clearly, this kind of system behavior prevents that fitness apps on the smart phones can be used instantly, or at least quickly with only short preparation time.

\subsection{Review of Handling Fitness Tracking Apps on Smartphones}

The investigation here focuses on those smartphones, which are operated under the current market leader, the so-called Android operating system (statcounter, 2019). The official smartphone application software distribution source is operated by the Android project holder in a market place on the Web, which offers almost uncountable app tools for tracking in particular fitness activities of running and walking. For serving both categories, considerably more than 100 apps are available each, which can be sub-divided into those, where monetary costs apply for using the fully functional versions, while the others fall into the category of freeware in the sense, that no monetary costs are applied for all version levels. 
Defining as handling reference wearable sports computers for endurance workouts like running, walking and cycling (like unit $\mathrm{T}$ in Figure 1b), the standard for instant use can be described as follows: After switching on - regardless of first or repeated use -, the system is available for immdiate use with pre-configured settings and display arrangements. The only delay, which is invoked by modern sports computers is, that the system needs some time for its alignment to the satellite navigation signal. After localization precision is reached, most computers can immediately be started with one single UI action (e.g. a hard or soft button press). Pausing or finalizing a workout appears as simple. In summary, instant use of the devices applies possible in every days use; some devices may require few setup information during first use, which is typically requested from the user by a very short guided process, where information has to be entered like display language and possibly some physiological parameters of the training person.

In clear contradiction to this kind of instant usability, handling of smartphone apps for endurance fitness workouts requires in general many stages more. Starting-up for the very first time with a particular app consists of the following handling steps (in the work here, this was practically experienced with a broader set of fitness apps like shown in Figure 3, and can also be logically deducted from the standard app programming concepts on smartphones):

- Analysis of available apps in the market place

- If not yet available, register account for market place

- Download and install particular app

- Revoke access rights of the app

- Place app launch on top UI level

- Start app

- Register new account for use of the app or link to a social network account

- Enter physiological information of the training person and possibly other personal data

- Select specific workout mode, in some cases from an extremely long selection list

- Await availability of localization system, since this is usually deactivated for energy saving reasons before this handling point

More configuration steps or other actions like de-selecting irrelevant presentation contents like commercial advertisements may be required additionally, before a first test of the tracking app renders possible.

In the case of daily use in workouts - when the first use has been successfully performed before - the following UI stages are required typically (also as practically validated in handling tests here):

- Unlock smartphone screen from sleeping mode

- Manually launch fitness app

- Select in a sub-menu the desired activity and display mode

- Start recording, but reasonable only after waiting time for positioning sensor precision

- Stop activity by simple UI actions

- Leaving the app usually requires to enter additional information for saving the trace

As further aspects it can be observed, that UI screens in such apps tend to be overloaded with information details, which leads to small text display and tiny input elements, which are especially not feasible for elder user with reduced haptic skills and difficulties in reading close-by surfaces. The arrangement of the UI elements also do not appear thoroughly elaborated in many app samples (Figure 3).

As intermediate result, it can be stated at this point, that the handling of the smartphones apps requires long series of tiny actions, therefore, the feasibility of such fitness apps for instant use has to be neglected. Furthermore, the readability, the clearness of the information presentation does not meet established standards of UI programming.

Few apps could be identified, which are offering a simple-styled UI with bigger text displays and less UI hierarchy. Unfortunately, such apps turned out being unstable, which leaded to a loss of workout trace because the app tool killed itself unexpectedly (middle app in Figure 3). Another observation is, that also popular and widely distributed apps for runners use to show sometimes spurious effects like displaying the just conducted running route through massive buildings. 


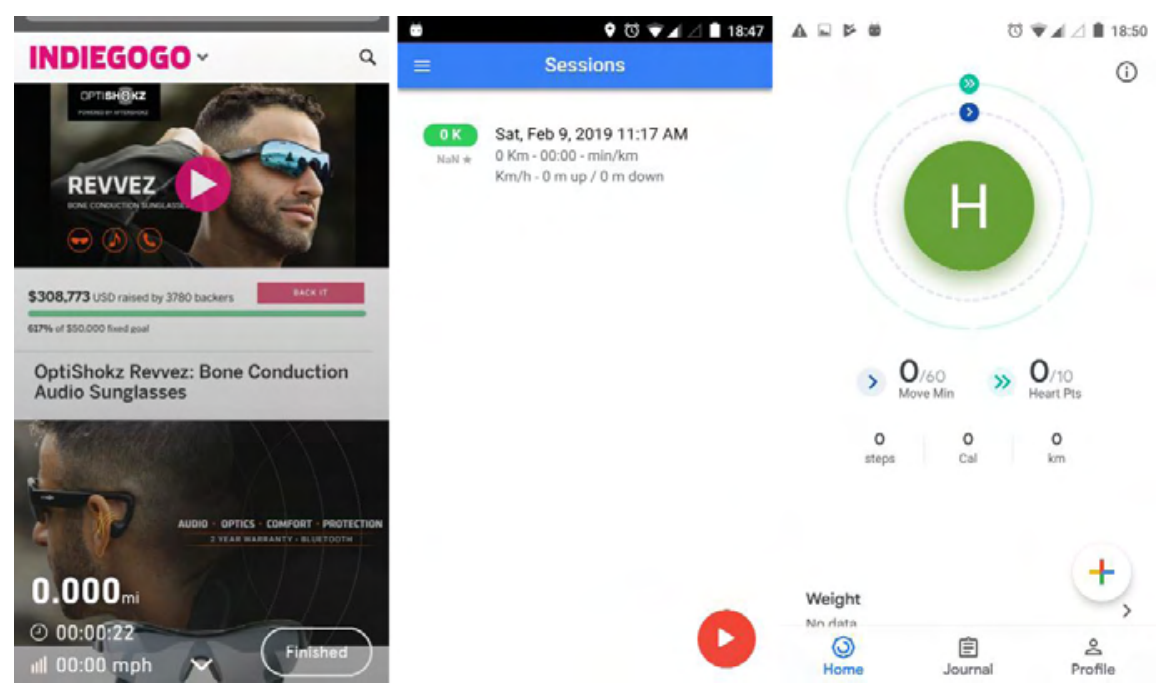

Figure 3. Screenshots of different fitness apps: on the left a "charity" app is shown, which spams the user not only by e-mails to the registered account, but also the activity display screen here is spoiled by commercial ads; the middle app is styled simplistic, but displays $\mathrm{NaN}$ as result of wrong internal processing and uses to kill itself, while the activity of the fitness app provided by the OS vendor is totally unclear in its controls, and uses - as all others - tiny text fonts and wastes most of the available screen surface

Concerning the needs of elderly users, it is also visible already in the few samples here (Figure 3 ) - and this was also the strict misfit in all other tested apps - that handling problems like inefficient or unintentional (multi)-touching in UI input as elements indicated early (Harada et al., 2013), is not served at all. Same finding applies for guiding through consecutive UI screen arrangements with missing clearly visible controls for canceling or back-navigation. The limited readability of the screen contents, which was discussed before, will render even more adverse for people with eyesight impairments (Adiseshiah, 2017).

\section{CRITICAL DISCUSSION}

The handling of specialized sports computers in endurance workouts like $T$, which are usually available in the housing of wrist watches, is well established for a long while already. Since such devices are intended for a specific purpose, they are properly pre-configured already directly out of the selling box for a first use; they are in general ready to use even more directly after power-on later in daily operation. Only waiting time applies for launching the satellite positioning, which is today used outdoor as mandatory standard. Though the displays of such devices have got small dimensions, they are optimized for readability in day light outdoor, and they are arranged clearly with big letters. The users can - for most devices - even assemble the desired screen components and by that influence also the readability according to best personal desires.

Within the many smartphones fitness tracking apps, the user is confronted with totally different demands. In most cases, at first a lengthy registration with common or proprietary social (sports) networks is required, and the user is forced to deliver lots of personal data. Other information may be collected by the app without giving clear notification about the extreme data thirst of the app, which in most cases has got no relation to the indented use scenario. Extreme samples are access to the entire phone book and contact lists of the sports app user or even the demand of issuing calls and messaging over the phone network.

In the end, the user is enabled only after a lengthy setup process to use the app for the desired purpose; using it again later for the same purpose usually also arises as complicate. Some apps - e.g. the fitness app distributed by the operating system vendor of the devices here - offer meanwhile an extremely long list of possible activities, which can be tracked and recorded. The selection among these requires another UI entering stage before the app can be used in the desired way. Furthermore, the screen arrangements do not really follow any reasonable UI concepts (Figure 3). It must be assumed that programmers in this market are developing tools, while they themselves don't have any experience or idea, what would be really required in using them efficiently. 


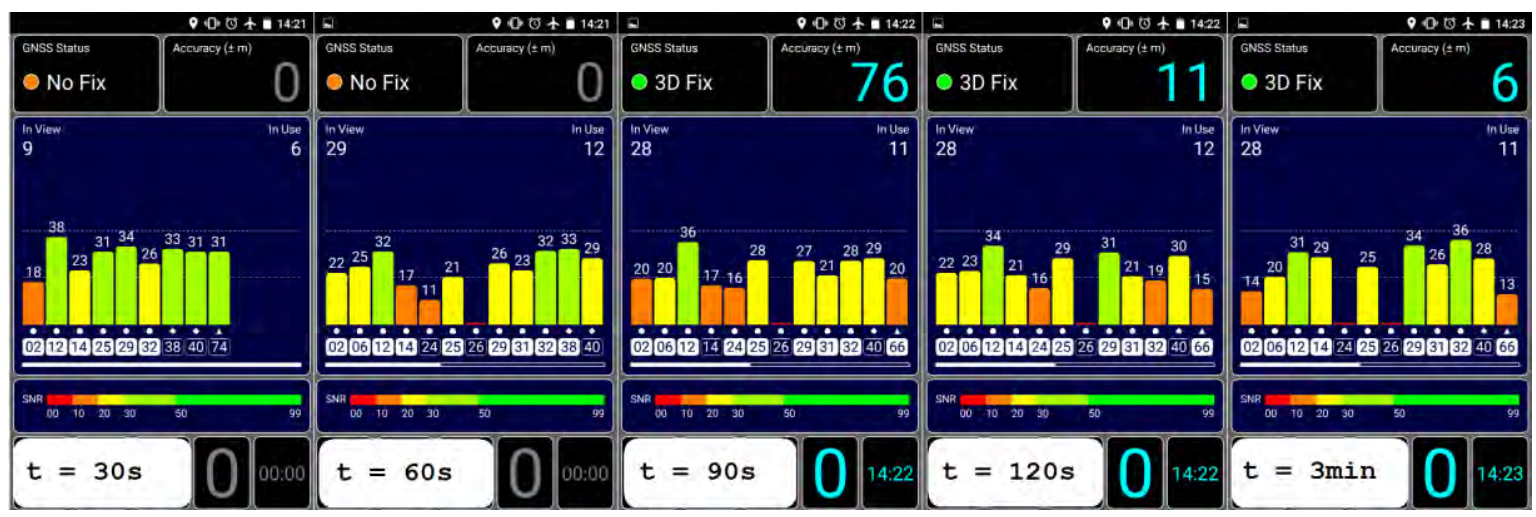

Figure 4. Set of consecutive display screens after launching the GPS analysis app and by that starting the localization sensing on unit $\mathrm{B}$. The imprints at the bottom are listing the screen capturing times, and the corresponding positioning accuracy is displayed in the upper right corner as measure in meters. Accordingly, tracking is not possible in the first minute at all, and it requires three minutes for reaching a usable - but not good - precision of 6 meters

Some further fundamental restriction arises from the localization technologies used in smartphones. GPS usually is de-activated for saving energy consumption in this systems, but when it is launched only with the fitness app, it incurs an extended waiting time at this point of handling (Figure 4). Up to several minutes delay, which often applies, makes the entire tool worthless. Another effect disturbs this breaking effect even more: localization improvement in the underlying operating system mechanisms exploits additional information from other networks like the cellular phone system or from WLAN access points with the aim of improving and speeding up localization. This all uses to fail easily in outdoor environments, when no known networks are reachable or close by. It had to be observed, that in such situations some fitness apps refuse to work at all, if the training is started in a forest or a rural area, instead close to a city center with all its different digital RF networks.

In summary it has to be concluded, that actually available smartphone fitness apps are no prospective candidates for replacing the wearable endurance sport computers, since they do neither offer seamless, nor clear, nor instant usability.

\section{CONCLUSION}

As validated in the practical experiments here, recent generation smartphones are representing a feasible host base, which consists of a hardware platform with an operational software environment, for executing potentially reliable tracking tools for physical health and sports activities. Unfortunately, among a broad mass of software apps, no instant or easy-to-use solution is available for daily endurance workouts like health brisk walking or running.

Theoretically, such apps could be developed quite straight forwardly, but in the end there will always remain a last level of handling inconvenience, because smartphones usually hold simultaneously a mass of apps for quite different purposes; therefore, any tracking app has to be always launched manually from a broader choice before, and the device may not be ready or well prepared for the fitness tracking - especially sensors usually are not fully operational -, which then incurs further delay before efficient use.

On the other hand, the major issue appears today being the primary intent of the fitness app programmers, who are more focusing on collecting personal data and behavior patterns of the user, instead on aiming for an efficient, easy and clear handling of their app as tool for workout tracking. The latter would be even more important in supporting health activities of elderly with limited haptic capabilities and restricted eyesight.

Accordingly at the current market situation, monitoring units like specialized sports watches or personal medical monitors are representing the only solution, which yields seamless and instant use for daily physical workouts in endurance sports and health applications. As validated here, commercial smartphones could serve as sufficient hardware and software base as well, but appropriate apps are not available yet. 


\section{REFERENCES}

Abbott, R.D et al., 1994. Physical activity in older middle-aged men and reduced risk of stroke: the Honolulu Heart Program. Am. J. Epidemiol., Vol. 139(9), pp 881-893.

Adiseshiah, E.G., 2017. UX Design Thinking from A Senior Citizen's Perspective, UsabilityGeek, https://usabilitygeek.com/ux-design-thinking-senior-citizen-user/, last accessed 2019/03/20

Alsulami, M. H., Atkins, A. S., 2016. A model of a proactive ambient assisted living system to monitor elderly people in the Kingdom of Saudi Arabian. IJRET, vol. 4, no. 1 (March 2016), pp 35-41.

Church, T., 2010. Physical Activity and Heart Disease in Obesity, in: Physical Activity and Obesity, 2nd ed., Human Kinetics, Champaign (US), p282.

Colagiuri, S., et al., 2010. The cost of overweight and obesity in Australia. Med J Aust, 192, 5 (March 2010), pp 260-264.

Harada, S., et al., 2013 Characteristics of Elderly User Behavior on Mobile Multi-touch Devices, INTERACT 2013, Part IV, LNCS 8120, pp 323-341.

Jefferis, B.J. et al., 2014. Protective effect of time spent walking on risk of stroke in older men. Stroke, Vol. 45, pp 194-199.

Law, M.R. et al., 1991. Strategies for prevention of osteoporosis and hip fracture. British Medical Journal, Vol. 303, pp 453-459.

Oguma, Y., Shinoda-Tagawa, T., 2004. Physical activity decreases cardiovascular disease risk in women. Am. J. Prev. Med., Vol. 26(5), pp 407-418.

Schnohr, P. et al., 2015, Dose of Jogging and Long-Term Mortality: The Copenhagen City Heart Study, Journal of the American College of Cardiology, Vol. 65(5), pp 411-419.

statcounter, 2019. Globalstats, http://gs.statcounter.com/os-market-share/mobile/worldwide, last accessed 2019/03/18.

Suter, E. et al., 1994. Jogging or walking - Comparison of health effects. Annals of Epidemiology, Vol. 4(5), pp 375-381.

Valentín, G., Howard, A. M., 2013. Dealing with Childhood Obesity: Passive versus Active Activity Monitoring Approaches for Engaging Individuals in Exercise. In Proceedings BRC 2013 (Rio de Janeiro, Brazil, Feb. 2013).

Wannamethee, G, Shaper, A. G., 1992. Physical activity and stroke in British middle aged men. British Medical Journal, 304 (March 1992), pp597-601.

Weghorn, H., 2019, Unsubstantial Health and Sports Monitoring Reliability of Commercial Fitness Tracker Bracelets Induced by Their All-in-One Sensing Unit Approach - Experimental Evaluation of Measurement Accuracy in Dynamic and in Steady Physical Effort Scenarios, icSPORTS 2016/2017: Sport Science Research and Technology Support, CCIS, Vol. 975, Springer, Cham, pp 55-74. 